\title{
Special issue: Data visualization for decision-making: an important issue
}

\section{Corentin Burnay $^{1} \cdot$ Fatima Dargam ${ }^{2} \cdot$ Pascale Zarate $^{3}$}

Published online: 6 November 2019

(c) Springer-Verlag GmbH Germany, part of Springer Nature 2019

In contemporary society, most individuals, and especially leaders and managers in organizations, are confronted with complex decision problems on a daily basis. Decisions become complex when they are distributed and unstructured, so that a single decision maker relying solely on her own knowledge has little chances to solve it properly. This is true in various contexts, ranging from small private companies to large public institutions. Nowadays, the sustainability of business models and growth of organizations depend on their capability to make good decisions and ultimately to solve those highly complex problems.

To tackle the problem of complex decision problems, decision makers can rely on external sources of information, that will help them acquire necessary insight into the problem and collaborate more effectively in order to solve the problem. Various information systems can help decision makers in such endeavor. Information technologies such as Business Intelligence Systems, Big Data or Knowledge Management Systems are just three examples, among many others, of systems that gather data and produce information to support decision makers. These systems help decision makers understanding the business, its direct and indirect environment, helps in the assessment of the different trade-offs in the company, in the estimation of the costs associated with the different decision alternatives, and can ultimately help people to optimize their decision process as well as its final outcome.

Methods and strategies used in those different types of systems all share an important issue to deal with, that is the one of bringing up an adequate and comprehensible visualization approach to present relevant information to users and decisionmakers in an effective way. This special issue collects some recent approaches of Decision Support Systems and of Information Systems that present domain-specific

Corentin Burnay

corentin.burnay@unamur.be

1 Département de gestion, Université de Namur, 8 rue rempart de la vierge, 5000 Namur, Belgium

2 SimTech Simulation Technology, Riesstrasse 120, Graz, Austria

3 Faculté d'Informatique - IRIT, Université Toulouse 1 Capitole, 2 rue du Doyen Gabriel Marty, 31042 Toulouse Cedex 9, France 
and visualization-based user communication strategies and interfaces to support decision-making.

A first paper entitled "Spatial Visualization on Patterns of Disaggregate Robberies" by Nepomuceno and Cabral Seixas Costa starts from the observation that many studies propose visual representations of aggregated spatial criminal data, which fail to provide sufficient insight to understand criminality issues in a given city. In this paper, the author decomposes a large dataset of robbery incidents into 9 smaller sets of specific robberies and propose a new type of visualization in order to support decision making regarding public safety resources.

A second contribution in this special issue proposes an innovative perspective for event analytics and is entitled "Visualizing and exploring event databases: A methodology to benefit from Process Analytics". In this paper, Delias, Zoumpoulidis and Kazanidis present a process-based analysis of event data and propose a methodology to conduct such analysis. Event databases provide a wealth of interesting information but are not easy to explore and performing knowledge discovery is a very complex process that is discussed in this paper.

A third paper goes on a totally different type of data, illustrating the variety of problem to be dealt with when it comes to data visualization. In their contribution entitled "Using Data Visualization for Ranking Alternatives with Partial Information and Interactive Tradeoff", Asfora Frej, Teixeira de Almeida and Cabral Seixas Costa deal with ranking visualization, and more precisely with data visualization within a method for solving multi-criteria decision-making (MCDM) problems for ranking of alternatives, based on flexible and interactive tradeoff (the so-called FITradeoff) elicitation. They suggest a new approach to such ranking problem and come up with a new ranking visualization. In the same line of thinking, the paper "Decision Neuroscience for Improving Data Visualization of Decision Support in the FITradeoff Method" by Reis Peixoto Roselli, Teixeira de Almeida and Asfora Frej presents various neuroscience experiments on postgraduate students with the double objective of improving the FIT DSS methodology for eliciting MCDM/A weights and to help analysts in improving the use of different graphs in such approaches. Also related to MCDM, Kunsch and Brands propose a fifth paper entitled "Visualising multi-criteria weight elicitation by multiple stakeholders in complex decision systems" where they propose a technique to elicit weight from multiple stakeholders in a way that is more transparent. The intention of authors is to help in the design of complex systems, such as adaptive systems.

A sixth paper entitled "Where will the next ski injury occur? A system for visual and predictive analytics of ski injuries" is proposed by Radovanović, Delibašić, Suknović and Matović. In this paper, authors propose a model for predicting ski injuries, together with a model-development methodology. Predictions discussed in this paper are spatial and temporal, and are carried out in near real time. The model is implemented in a system that offers a visual decision support to ski-resort medical teams.

A last contribution by Wachowicz, Kersten and Roszkowska, entitled "How Do I Tell You What I Want? Agent's Interpretation of Principal's Preferences and Its Impact on Understanding the Negotiation Process and Outcomes" investigates a visualization issue in the context of principal-agent problems. Authors analyze the 
way principal's preferences are visualized, and how such representation may affect the accuracy of representation of this principal by its agent. Authors conclude their work with some practical recommendations about the representation and elicitation of preferences in principal-agent problems.

Altogether, the selected papers demonstrate the variety and significance of issues to be dealt with in the field of decision making and data visualization. Producing a graph or a map and plotting data on it is one thing, but designing visualizations that actually support decision makers is something completely different which is far from being trivial. This special issue demonstrates this very clearly, and opens the door for many new researches.

Publisher's Note Springer Nature remains neutral with regard to jurisdictional claims in published maps and institutional affiliations. 